\title{
Oscillation criteria for second-order nonlinear neutral difference equations of mixed type
}

\author{
Ethiraju Thandapani ${ }^{* *}$, Nagabhushanam Kavitha ${ }^{1}$ and Sandra Pinelas ${ }^{2}$
}

\author{
* Correspondence: \\ ethandapani@yahoo.co.in \\ ${ }^{1}$ Ramanujan Institute for Advanced \\ Study in Mathematics, University of \\ Madras, Chennai 600 005, India \\ Full list of author information is \\ available at the end of the article
}

\begin{abstract}
Some oscillation criteria are established for the second order nonlinear neutral difference equations of mixed type.

$$
\Delta^{2}\left(x_{n}+a x_{n-\tau_{1}} \pm b x_{n+\tau_{2}}\right)^{\alpha}=q_{n} x_{n-\sigma_{1}}^{\beta}+p_{n} x_{x+\sigma_{2}}^{\beta}, n \geq n_{0}
$$

where $\alpha$ and $\beta$ are ratio of odd positive integers with $\beta \geq 1$. Results obtained here generalize some of the results given in the literature. Examples are provided to illustrate the main results.
\end{abstract}

2010 Mathematics Subject classification: 39A10.

Keywords: Neutral difference equation, mixed type, comparison theorems, oscillation.

\section{Introduction}

In this article, we study the oscillation behavior of solutions of mixed type neutral difference equation of the form,

$$
\Delta^{2}\left(x_{n}+a x_{n-\tau_{1}} \pm b x_{n+\tau_{2}}\right)^{\alpha}=q_{n} x_{n-\sigma_{1}}^{\beta}+p_{n} x_{x+\sigma_{2}}^{\beta}
$$

where $n \in \mathbb{N}\left(n_{0}\right)=\left\{n_{0}, n_{0}+1, \ldots\right\}, n_{0}$ is a nonnegative integer, $a, b$ are real nonnegative constants, $\tau_{1}, \tau_{2}, \sigma_{1}$, and $\sigma_{2}$ are positive integers, $\left\{q_{n}\right\}$ and $\left\{p_{n}\right\}$ are positive real sequences and $\alpha, \beta$ are ratio of odd positive integers with $\beta \geq 1$.

Let $\theta=\max \left\{\tau_{1}, \sigma_{1}\right\}$. By a solution of Equation $\left(E_{ \pm}\right)$we mean a real sequence $\left\{x_{n}\right\}$ which is defined for $n \geq n_{0}-\theta$ and satisfies Equation $\left(E_{ \pm}\right)$for all $n \in \mathbb{N}\left(n_{0}\right)$. A nontrivial solution of Equation $\left(E_{ \pm}\right)$is said to be oscillatory if it is neither eventually positive nor eventually negative. Otherwise it is known as nonoscillatory.

Equations of this type arise in a number of important applications such as problems in population dynamics when maturation and gestation are included, in cobweb models, in economics where demand depends on the price at an earlier time and in electric networks containing lossless transmission lines. Hence it is important and useful to study the oscillation behavior of solutions of neutral type difference Equation $\left(E_{ \pm}\right)$.

The oscillation, nonoscillation and asymptotic behavior of solutions of Equation $\left(E_{ \pm}\right)$, when $b=0$ and $p_{n} \equiv 0$ or $a=0$ and $p_{n} \equiv 0$ or $b=0$ and $q_{n} \equiv 0$ have been considered by many authors, see for example [1-4] and the reference cited therein. However, there are few results available in the literature regarding the oscillatory properties of neutral difference equations of mixed type, see for example [1-8]. Motivated by the above 
observation, in this article we establish some new oscillation criteria for the Equation $\left(E_{ \pm}\right)$which generalize some of the results obtained in [1-3,5-7].

In Section 2, we present conditions for the oscillation of all solutions of equation ( $E$ ${ }_{ \pm}$). Examples are provided in Section 3 to illustrate the results.

\section{Oscillation results}

In this section, we obtain sufficient conditions for the oscillation of all solutions of Equation $\left(E_{ \pm}\right)$. First we consider the Equation $\left(E_{-}\right)$, viz,

$$
\Delta^{2}\left(x_{n}+a x_{n-\tau_{1}}-b x_{n+\tau_{2}}\right)^{\alpha}=q_{n} x_{n-\sigma_{1}}^{\beta}+p_{n} x_{x+\sigma_{2}}^{\beta} \quad n \in \mathbb{N}\left(n_{0}\right) .
$$

To prove our main results we need the following lemma, which can be found in [9].

Lemma 2.1. Let $A \geq 0, B \geq 0$ and $\gamma \geq 1$. Then

$$
A^{\gamma}+B^{\gamma} \geq \frac{1}{2^{\gamma-1}}(A+B)^{\gamma}
$$

and

$$
A^{\gamma}-B^{\gamma} \geq(A-B)^{\gamma}, \text { if } A \geq B .
$$

Theorem 2.2. Let $\sigma_{1}>\tau_{1}, \sigma_{2}>\tau_{2}$ and $\left\{q_{n}\right\}$ and $\left\{p_{n}\right\}$ are positive real nonincreasing sequences. Assume that the difference inequalities

i)

$$
\Delta^{2} y_{n}-\frac{p_{n}}{2^{\beta-1}\left(1+a^{\beta}\right)^{\beta / \alpha}} y_{n+\sigma_{2}}^{\beta / \alpha} \geq 0
$$

has no eventually positive increasing solution,

ii)

$$
\Delta^{2} y_{n}-\frac{p_{n}}{2^{\beta-1}\left(1+a^{\beta}\right)^{\beta / \alpha}} y_{n-\sigma_{2}+\tau_{1}}^{\beta / \alpha} \geq 0
$$

has no eventually positive decreasing solution,

iii)

$$
\Delta^{2} y_{n}+\frac{q_{n}}{b^{\beta}} y_{n-\sigma_{1}-\tau_{2}}^{\beta / \alpha}+\frac{p_{n}}{b^{\beta}} y_{n+\sigma_{2}-\tau_{2}}^{\beta / \alpha} \leq 0
$$

has no eventually positive solution.

Then every solution of Equation $\left(E_{-}\right)$is oscillatory.

Proof. Let $\left\{x_{n}\right\}$ be a nonoscillatory solution of Equation $\left(E_{-}\right)$. Without loss of generality, we may assume that there exists $n_{1} \in \mathbb{N}\left(n_{0}\right)$ such that $x_{n-\theta}>0$ for all $n \geq n_{1}$. Set $z_{n}=\left(x_{n}+a x_{n-\tau_{1}}-b x_{n+\tau_{2}}\right)^{\alpha}$.

Then

$$
\Delta^{2} z_{n}=q_{n} x_{n-\sigma_{1}}^{\beta}+p_{n} x_{n+\sigma_{2}}^{\beta}>0, \quad n \geq n_{1},
$$


which implies that $\left\{z_{n}\right\}$ and $\left\{\Delta z_{n}\right\}$ are of one sign for all $n_{2} \geq n_{1}$. We claim that $z_{n}>0$ eventually. To prove it assume that $z_{n}<0$. Then we let

$$
0<u_{n}=-z_{n}=\left(b x_{n+\tau_{2}}-a x_{n-\tau_{1}}-x_{n}\right)^{\alpha} \leq b^{\alpha} x_{n+\tau_{2}}^{\alpha} .
$$

Thus

$$
x_{n}^{\beta} \geq \frac{1}{b^{\beta}} u_{n-\tau_{2}}^{\beta / \alpha} \quad n \geq n_{2}
$$

From Equation $\left(E_{-}\right)$, we get

$$
0=\Delta^{2} u_{n}+q_{n} x_{n-\sigma_{1}}^{\beta}+p_{x} x_{n+\sigma_{2}}^{\beta} \geq \Delta^{2} u_{n}+\frac{q_{n}}{b^{\beta}} u_{n-\sigma_{1}-\tau_{2}}^{\beta / \alpha}+\frac{p_{n}}{b^{\beta}} u_{n+\sigma_{2}-\tau_{2}}^{\beta / \alpha} .
$$

Hence $\left\{u_{n}\right\}$ is a positive solution of inequality (2.5), a contradiction.

Therefore $z_{n} \geq 0$. We define

$$
y_{n}=z_{n}+a^{\beta} z_{n-\tau_{1}}-\frac{b^{\beta}}{2^{\beta-1}} z_{n+\tau_{2}} .
$$

Then, we have

$$
\begin{aligned}
\Delta^{2} y_{n}= & \Delta^{2} z_{n}+a^{\beta} \Delta^{2} z_{n-\tau_{1}}-\frac{b^{\beta}}{2^{\beta-1}} \Delta^{2} z_{n+\tau_{2}} \\
= & q_{n} x_{n-\sigma_{1}}^{\beta}+p_{n} x_{n+\sigma_{2}}^{\beta}+a^{\beta} q_{n-\tau_{1}} x_{n-\sigma_{1}-\tau_{1}}^{\beta}+a^{\beta} p_{n-\tau_{1}} x_{n+\sigma_{2}-\tau_{1}}^{\beta} \\
& -\frac{b^{\beta}}{2^{\beta-1}} q_{n+\tau_{2}} x_{n-\sigma_{1}+\tau_{2}}^{\beta}-\frac{b^{\beta}}{2^{\beta-1}} p_{n+\tau_{2}} x_{n+\sigma_{2}+\tau_{2}}^{\beta} .
\end{aligned}
$$

Using the inequality (2.1) in (2.7), we obtain

$$
\begin{aligned}
\Delta^{2} y_{n} \geq & \frac{q_{n}}{2^{\beta-1}}\left(x_{n-\sigma_{1}}+a x_{n-\sigma_{1}-\tau_{1}}\right)^{\beta}-\frac{b^{\beta}}{2^{\beta-1}} q_{n+\tau_{2}} x_{n-\sigma_{1}+\tau_{2}}^{\beta} \\
& +\frac{p_{n}}{2^{\beta-1}}\left(x_{n+\sigma_{2}}+a x_{n+\sigma_{2}-\tau_{1}}\right)^{\beta}-\frac{b^{\beta}}{2^{\beta-1}} p_{n+\tau_{2}} x_{n+\sigma_{2}+\tau_{2}}^{\beta}
\end{aligned}
$$

Now using the inequality (2.2), we obtain

$$
\Delta^{2} y_{n} \geq \frac{q_{n}}{2^{\beta-1}} z_{n-\sigma_{1}}^{\beta / \alpha}+\frac{p_{n}}{2^{\beta-1}} z_{n+\sigma_{2}}^{\beta / \alpha}>0
$$

Consequently $\left\{y_{n}\right\}$ and $\left\{\Delta y_{n}\right\}$ are of one sign, eventually. Now we shall prove that $y_{n}>$ 0 . If not, then let

$$
0<v_{n}=-y_{n}=\frac{b^{\beta}}{2^{\beta-1}} z_{n+\tau_{2}}-a^{\beta} z_{n-\tau_{1}}-z_{n} \leq \frac{b^{\beta}}{2^{\beta-1}} z_{n+\tau_{2}} .
$$

Hence

$$
z_{n} \geq \frac{2^{\beta-1}}{b^{\beta}} v_{n-\tau_{2}}
$$

and (2.8) implies

$$
0 \geq \Delta^{2} v_{n}+\frac{q_{n}}{b^{\beta}} v_{n-\sigma_{1}-\tau_{2}}^{\beta / \alpha}+\frac{p_{n}}{b^{\beta}} v_{n+\sigma_{2}-\tau_{2}}^{\beta / \alpha} .
$$


We obtain that $\left\{v_{n}\right\}$ is a positive solution of inequality (2.5), a contradiction. Next we consider the following two cases:

Case 1: Let $\Delta z_{n}<0$ for $n \geq n_{3} \geq n_{2}$. We claim that $\Delta y_{n}<0$ for $n \geq n_{3}$. If not, then we have $y_{n}>0, \Delta y_{n}>0$ and $\Delta^{2} y_{n} \geq 0$ which implies that $\lim _{n \rightarrow \infty} y_{n}=\infty$. On the other hand, $z_{n}>0, \Delta z_{n}<0$ implies that $\lim _{n \rightarrow \infty} z_{n}=c<\infty$. Then applying limits on both sides of (2.6) we obtain a contradiction. Thus $\Delta y_{n}<0$ for $n \geq n_{3}$. Using the monotonicity of $\left\{z_{n}\right\}$, we now get

$$
y_{n-\sigma_{1}}=z_{n-\sigma_{1}}+a^{\beta} z_{n-\sigma_{1}-\tau_{1}}-\frac{b^{\beta}}{2^{\beta-1}} z_{n-\sigma_{1}+\tau_{2}} \leq\left(1+a^{\beta}\right) z_{n-\sigma_{1}-\tau_{1}} .
$$

This together with (2.8) implies

$$
\Delta^{2} y_{n} \geq \frac{q_{n}}{2^{\beta-1}\left(1+a^{\beta}\right)^{\beta / \alpha}} y_{n-\sigma_{1}+\tau_{1}}^{\beta / \alpha} .
$$

Thus $\left\{y_{n}\right\}$ is a positive decreasing solution of inequality (2.4), a contradiction. Case 2: Let $\Delta z_{n}>0$ for $n \geq n_{3}$. Now we consider the following two cases. Case (i): Assume that $\Delta y_{n}<0$ for $n \geq n_{3}$. Proceeding similarly as above and using the monotonicity of $\left\{z_{n}\right\}$ we obtain

$$
y_{n-\sigma_{1}} \leq\left(1+a^{\beta}\right) z_{n-\sigma_{1}} .
$$

Then using this in (2.8) we obtain

$$
\Delta^{2} y_{n} \geq \frac{q_{n}}{2^{\beta-1}} z_{n-\sigma_{1}}^{\beta / \alpha} \geq \frac{q_{n}}{2^{\beta-1}\left(1+a^{\beta}\right)^{\beta / \alpha}} y_{n-\sigma_{1}}^{\beta / \alpha} \geq \frac{q_{n}}{2^{\beta-1}\left(1+a^{\beta}\right)^{\beta / \alpha}} y_{n-\sigma_{1}+\tau_{1}}^{\beta / \alpha}
$$

and again $\left\{y_{n}\right\}$ is a positive decreasing solution of inequality (2.4), a contradiction.

Case (ii): Assume that $\Delta y_{n}>0$ for $n \geq n_{3}$. Then $y_{n+\sigma_{2}} \leq\left(1+a^{\beta}\right) z_{n+\sigma_{2}}$ which in view of (2.8) implies

$$
\Delta^{2} y_{n} \geq \frac{p_{n}}{2^{\beta-1}} z_{n+\sigma_{2}}^{\beta / \alpha} \geq \frac{p_{n}}{2^{\beta-1}\left(1+a^{\beta}\right)^{\beta / \alpha}} y_{n+\sigma_{2}}^{\beta / \alpha}
$$

that is, (2.3) has a positive increasing solution, a contradiction. The proof is complete.

Remark 2.1. Theorem 2.2 permits us to obtain various oscillation criteria for Equation (E.). Moreover we are able to study the asymptotic properties of solutions of Equation $\left(E_{-}\right)$even if not all assumptions of Theorem 2.2 are satisfied. If the difference inequality (2.3) has an eventually positive increasing solution then the conclusion of Theorem 2.2 is replaced by "Every solution of Equation $\left(E_{-}\right)$is either oscillatory or $\left|x_{n}\right|$ $\rightarrow \infty$ as $n \rightarrow \infty$ ".

Remark 2.2. In [2, Theorem 7.6.26], the author considered the Equation (E-) with $\alpha=$ $\beta=1, p_{n} \equiv p$, and $q_{n} \equiv q$ and obtain oscillation results with $(1+a-b)>0$. Hence Theorem 2.2 generalize and improve the results of [2, Theorem 7.6.26].

Remark 2.3. Applying existing conditions sufficient for the inequalities (2.3), (2.4), and (2.5) to have no above mentioned solutions, we immediately obtain various oscillation criteria for Equation $\left(E_{-}\right)$. 
Theorem 2.3. Let $\sigma_{1}>\tau_{1}, \sigma_{2} \geq 2$, and $\beta=\alpha$. Assume that

$$
\limsup _{n \rightarrow \infty} \sum_{s=n}^{n+\sigma_{2}-\tau_{2}}\left(n+\sigma_{2}-s-1\right) p_{s}>\left(1+a^{\alpha}\right) 2^{\alpha-1}
$$

and

$$
\limsup _{n \rightarrow \infty} \sum_{s=n-\sigma_{1}+\tau_{1}}^{n}\left(n-s+\sigma_{1}-\tau_{1}+1\right) q_{s}>\left(1+a^{\alpha}\right) 2^{\alpha-1}
$$

and that the difference inequality (2.5) has no eventually positive solution. Then every solution of Equation $\left(E_{-}\right)$is oscillatory.

Proof. Conditions (2.9) and (2.10) are sufficient for the inequality (2.3) to have no increasing positive solution and for (2.4) to have no decreasing positive solution, respectively (see e.g., [2, Lemma 7.6.15]). The proof then follows from Theorem 2.2.

Remark 2.4. Taking into account the result of [2], we see that the absence of positive solution of (2.5) can be replaced by the assumption that for the corresponding equation

$$
\Delta^{2} y_{n}+\frac{q_{n}}{b^{\beta}} y_{n-\sigma_{1}-\tau_{2}}^{\beta / \alpha}+\frac{p_{n}}{b^{\beta}} y_{n+\sigma_{2}-\tau_{2}}^{\beta / \alpha}=0
$$

every solution of this equation are oscillatory.

Next we consider the difference Equation $\left(E_{+}\right)$

$$
\Delta^{2}\left(x_{n}+a x_{n-\tau_{1}}+b x_{n+\tau_{2}}\right)^{\alpha}=q_{n} x_{n-\sigma_{1}}^{\beta}+p_{n} x_{n+\sigma_{2}}^{\beta}
$$

and present conditions for the oscillation of all solutions of Equation $\left(E_{+}\right)$.

Theorem 2.4. Assume that $\sigma_{1} \geq \tau_{1}, \sigma_{2} \geq \tau_{2}+2, q_{n}^{*}=\min \left\{q_{n-\sigma_{1}}, q_{n}, q_{n+\tau_{2}}\right\}$ and $p_{n}^{*}=\min \left\{p_{n-\sigma_{1}}, p_{n}, p_{n+\tau_{2}}\right\}$. If

$$
\Delta^{2} y_{n}-\frac{p_{n}^{*}}{4^{\beta-1}\left(1+a^{\beta}+\frac{b^{\beta}}{2^{\beta-1}}\right)^{\beta / \alpha}} y_{n-\tau_{2}+\sigma_{2}}^{\beta / \alpha} \geq 0 .
$$

has no eventually positive increasing solution, and

$$
\Delta^{2} y_{n}-\frac{p_{n}^{*}}{4^{\beta-1}\left(1+a^{\beta}+\frac{b^{\beta}}{2^{\beta-1}}\right)^{\beta / \alpha}} \gamma_{n-\sigma_{2}+\tau_{2}}^{\beta / \alpha} \geq 0,
$$

has no eventually positive decreasing solution, then every solution of $\left(E_{+}\right)$is oscillatory. Proof. Let $\left\{x_{n}\right\}$ be a nonoscillatory solution of $\left(E_{+}\right)$. Without loss of generality, we assume that there exists an integer $n_{1} \in \mathbb{N}\left(n_{0}\right)$ such that $x_{n-\theta}>0$ for all $n \geq n_{1}$. Setting

$$
z_{n}=\left(x_{n}+a x_{n-\tau_{1}}+b x_{n+\tau_{2}}\right)^{\alpha}
$$

and

$$
y_{n}=z_{n}+a^{\beta} z_{n-\tau_{1}}+\frac{b^{\beta}}{2^{\beta-1}} z_{n+\tau_{2}}
$$


Then $z_{n}>0, y_{n}>0$ and

$$
\Delta^{2} z_{n}=q_{n} x_{n-\sigma_{1}}^{\beta}+p_{n} x_{n+\sigma_{2}}^{\beta} \geq 0 .
$$

Then $\left\{\Delta z_{n}\right\}$ is of one sign, eventually. On the other hand

$$
\begin{aligned}
\Delta^{2} y_{n}= & q_{n} x_{n-\sigma_{1}}^{\beta}+p_{n} x_{n+\sigma_{2}}^{\beta}+a^{\beta} q_{n-\tau_{1}} x_{n-\sigma_{1}-\tau_{1}}^{\beta}+a^{\beta} p_{n-\tau_{1}} x_{n+\sigma_{2}-\tau_{1}}^{\beta} \\
& +\frac{b^{\beta}}{2^{\beta-1}} q_{n+\tau_{2}} x_{n-\sigma_{1}+\tau_{2}}^{\beta}+\frac{b^{\beta}}{2^{\beta-1}} p_{n+\tau_{2}} x_{n+\sigma_{2}+\tau_{2}}^{\beta} .
\end{aligned}
$$

Using (2.1) in (2.15) we obtain

$$
\begin{aligned}
\Delta^{2} y_{n} \geq & \frac{q_{n}^{*}}{2^{\beta-1}}\left(x_{n-\sigma_{1}}+a x_{n-\sigma_{1}-\tau_{1}}\right)^{\beta}+\frac{q_{n+\tau_{2}}}{2^{\beta-1}} b^{\beta} x_{n-\sigma_{1}+\tau_{2}}^{\beta} \\
& +\frac{p_{n}^{*}}{2^{\beta-1}}\left(x_{n+\sigma_{2}}+a x_{n+\sigma_{2}-\tau_{1}}\right)^{\beta}+\frac{p_{n+\tau_{2}}}{2^{\beta-1}} b^{\beta} x_{n+\sigma_{2}+\tau_{2}}^{\beta}
\end{aligned}
$$

or

$$
\Delta^{2} y_{n} \geq \frac{1}{4^{\beta-1}}\left(q_{n}^{*} z_{n-\sigma_{1}}^{\beta / \alpha}+p_{n}^{*} z_{n+\sigma_{2}}^{\beta / \alpha}\right), \quad n \geq n_{1}
$$

Next we consider the following two cases:

Case 1: Assume that $\Delta z_{n}>0$. Then $\Delta y_{n}>0$. In view of (2.16), we have

$$
\Delta^{2} y_{n+\tau_{2}} \geq \frac{1}{4^{\beta-1}} p_{n+\tau_{2}}^{*} z_{n+\sigma_{2}+\tau_{2}}^{\beta / \alpha} .
$$

Applying the monotonicity of $z_{n}$, we find

$$
y_{n+\sigma_{2}}=z_{n+\sigma_{2}}+a^{\beta} z_{n-\tau_{1}+\sigma_{2}}+\frac{b^{\beta}}{2^{\beta-1}} z_{n+\tau_{2}+\sigma_{2}} \leq\left(1+a^{\beta}+\frac{b^{\beta}}{2^{\beta-1}}\right) z_{n+\tau_{2}+\sigma_{2}} .
$$

Combining (2.17) and (2.18) we have

$$
\Delta^{2} y_{n+\tau_{2}} \geq \frac{p_{n+\tau_{2}}^{*}}{4^{\beta-1}\left(1+a^{\beta}+\frac{b^{\beta}}{2^{\beta-1}}\right)^{\beta / \alpha}} y_{n+\sigma_{2}}^{\beta / \alpha} .
$$

Thus

$$
\Delta^{2} y_{n}-\frac{p_{n}^{*}}{4^{\beta-1}\left(1+\alpha^{\beta}+\frac{b^{\beta}}{2^{\beta-1}}\right)^{\beta / \alpha}} \gamma_{n-\tau_{2}+\sigma_{2}}^{\beta / \alpha} \geq 0 .
$$

Therefore $\left\{y_{n}\right\}$ is a positive increasing solution of the difference inequality (2.11), a contradiction.

Case 2: Assume that $\Delta z_{n}<0$. Then $\Delta y_{n}<0$. In view of (2.16) we see that

$$
\Delta^{2} y_{n-\tau 1} \geq \frac{1}{4^{\beta-1}} q_{n-\tau_{1}}^{*} z_{n-\tau_{1}-\sigma_{1}}^{\beta / \alpha} .
$$


From the monotonicity of $\left\{z_{n}\right\}$ we find

$$
y_{n-\sigma_{1}} \leq\left(1+a^{\beta}+\frac{b^{\beta}}{2^{\beta-1}}\right) z_{n-\tau_{1}-\sigma_{1}} .
$$

Combining the last two inequalities, we obtain

$$
\begin{aligned}
& \Delta^{2} y_{n-\tau_{1}} \geq \frac{q_{n-\tau_{1}}^{*}}{4^{\beta-1}\left(1+a^{\beta}+\frac{b^{\beta}}{2^{\beta-1}}\right)^{\beta / \alpha}} y_{n-\sigma_{1} .}^{\beta / \alpha} \\
& \Delta^{2} y_{n}-\frac{q_{n}^{*}}{4^{\beta-1}\left(1+a^{\beta}+\frac{b^{\beta}}{2^{\beta-1}}\right)^{\beta / \alpha}} y_{n-\sigma_{1}+\tau_{1}}^{\beta / \alpha} \geq 0 .
\end{aligned}
$$

Therefore $\left\{y_{n}\right\}$ is a positive decreasing solution of the difference inequality (2.12), a contradiction. This completes the proof.

Theorem 2.5. Assume that $\sigma_{1} \geq \tau_{1}, \sigma_{2} \geq \tau_{2}+2, \beta=\alpha, q_{n}^{*}=\min \left\{q_{n-\sigma_{1}}, q_{n}, q_{n+r 2}\right\}$ and $p_{n}^{*}=\min \left\{p_{n-\sigma_{1}}, p_{n}, p_{m+r 2}\right\}$. If

$$
\limsup _{n \rightarrow \infty} \sum_{s=n}^{n+\sigma_{2}-\tau_{2}-2}\left(n+\sigma_{2}-\tau_{2}-s-1\right) p_{s}^{*}>4^{\alpha-1}\left(1+a^{\alpha}+\frac{b^{\alpha}}{2^{\alpha-1}}\right),
$$

has no eventually positive increasing solution, and

$$
\limsup _{n \rightarrow \infty} \sum_{s=n-\sigma_{1}+\tau_{1}}^{n}(n-s+1) q_{s}^{*}>4^{\alpha-1}\left(1+a^{\alpha}+\frac{b^{\alpha}}{2^{\alpha-1}}\right)
$$

has no eventually positive decreasing solution, then every solution of $\left(E_{+}\right)$is oscillatory. Proof. Conditions (2.21) and (2.22) are sufficient for the inequality (2.11) to have no increasing positive solution and for (2.12) to have no decreasing positive solution, respectively (see e.g., [2, Lemma 7.6.15]). The proof then follows from Theorem 2.4.

Remark 2.5. When $a=\beta=1$, Theorem 2.5 involves result of Theorem 7.6 .6 of [2].

Theorem 2.6. Let $\beta=\alpha, \delta_{1}=\frac{\sigma_{1}-\tau_{1}}{2}>0$, and $\delta_{2}=\frac{\sigma_{2}-\tau_{2}}{2}>0$. Suppose that there exist two positive real sequence $\left\{\varphi_{n}\right\}$ and $\left\{\psi_{n}\right\}$ with $\Delta \varphi_{n} \geq 0$ and $\Delta \psi_{n} \leq 0$, such that

$$
q_{n}^{*} \geq 4^{\alpha-1}\left(1+a^{\alpha}+\frac{b^{a}}{2^{\alpha-1}}\right) \phi_{n+1} \phi_{n-\delta_{1}}
$$

and

$$
p_{n}^{*} \geq 4^{\alpha-1}\left(1+a^{\alpha}+\frac{b^{\alpha}}{2^{\alpha-1}}\right) \psi_{n+1} \psi_{n+\delta_{2}}
$$

where $p_{n}^{*}, q_{n}^{*}$ are as in Theorem 2.4. If the difference inequality

$$
\Delta v_{n}+\phi_{n-\delta_{1}} v_{n-\delta_{1}} \geq 0
$$


has no eventually negative solution, and

$$
\Delta v_{n}+\psi_{n-\delta_{2}} v_{n-\delta_{2}} \geq 0
$$

has no eventually positive solution, then every solution of Equation $\left(E_{+}\right)$is oscillatory.

Proof. Let $\left\{x_{n}\right\}$ be a nonoscillatory solution of Equation $\left(E_{+}\right)$. Without loss of generality, we assume that there exists an integer $n_{1} \in \mathbb{N}\left(n_{0}\right)$ such that $x_{n-\theta}>0$ for all $n \geq n_{1}$. Define $z_{n}$ and $y_{n}$ as in Theorem 2.4. Proceeding as in the proof of Theorem 2.4, we obtain (2.16). Next we consider the following two cases.

Case 1: Assume $\Delta z_{n}>0$. Clearly $\Delta y_{n}>0$. Then as in case 1 of Theorem 2.4, we find that $\left\{y_{n}\right\}$ is a positive increasing solution of inequality (2.19). Let $B_{n}-\Delta y_{n}+\psi_{n} y_{n+\delta_{2}}$. Then $B_{n}>0$. Using (2.24), we have

$$
\begin{aligned}
\Delta B_{n}-\frac{\Delta \psi_{n}}{\psi_{n}} B_{n}-\psi_{n+1} B_{n+\delta_{2}} & =\Delta^{2} y_{n}-\frac{\Delta \psi_{n}}{\psi_{n}} \Delta y_{n}-\psi_{n+1} \psi_{n+\delta_{2}} y_{n+2 \delta_{2}} \\
& \geq \Delta^{2} y_{n}-\psi_{n+1} \psi_{n+\delta_{2}} y_{n+2 \delta_{2}} \\
& \geq \Delta^{2} y_{n}-\frac{p_{n}^{*}}{4^{\alpha-1}\left(1+a^{\alpha}+\frac{b^{\alpha}}{2^{\alpha-1}}\right)} y_{n-\tau_{2}+\sigma_{2}} \\
& \geq 0 .
\end{aligned}
$$

Define $B_{n}=\psi_{n} v_{n}$. Then $\left\{v_{n}\right\}$ is a positive solution of (2.26), a contradiction. Case 2: Assume that $\Delta z_{n}<0$. Clearly $\Delta y_{n}<0$. Then as in case 2 of Theorem 2.4, we find that $\left\{y_{n}\right\}$ is a positive decreasing solution of inequality (2.20). Let $A_{n}-\Delta y_{n}+\phi_{n} y_{n-\delta_{1}}$. Then $A_{n}<0$. Using (2.23), we have

$$
\begin{aligned}
\Delta A_{n}-\frac{\Delta \phi_{n}}{\phi_{n}}-\phi_{n+1} A_{n-\delta_{1}} & =\Delta^{2} y_{n}-\frac{\Delta \phi_{n}}{\phi_{n}} \Delta y_{n}-\phi_{n+1} \phi_{n-\delta_{1}} y_{n-2 \delta_{1}} \\
& \geq \Delta^{2} y_{n}-\phi_{n+1} \phi_{n+\delta_{1}} y_{n-2 \delta_{1}} \\
& \geq \Delta^{2} y_{n}-\frac{q_{n}^{*}}{4^{\alpha-1}\left(1+a^{\alpha}+\frac{b^{\alpha}}{2^{\alpha-1}}\right)} y_{n-\sigma_{1}+\tau_{1}} \geq 0 .
\end{aligned}
$$

Define $A_{n}=\varphi_{n} v_{n}$. Then $\left\{v_{n}\right\}$ is a negative solution of inequality (2.25), a contradiction. This completes the proof.

From Theorem 2.6 and the results given in [7] we have the following oscillation criteria for Equation $\left(E_{+}\right)$.

Corollary 2.7. Let $\beta=\alpha, \delta_{1}=\frac{\sigma_{1}-\tau_{1}}{2}>0$, and $\delta_{2}=\frac{\sigma_{2}-\tau_{2}}{2}>0$. Suppose that there exist two positive real sequence $\left\{\varphi_{n}\right\}$ and $\left\{\psi_{n}\right\}$ with $\Delta \varphi_{n} \geq 0$ and $\Delta \psi_{n} \leq 0$ such that (2.23) and (2.24) holds. If

$$
\liminf _{n \rightarrow \infty} \sum_{s=n-\delta_{11}}^{n-1} \phi_{s-\delta_{1}}>\left(\frac{\delta_{1}}{\delta_{1}+1}\right)^{\delta_{1}+1}
$$

and

$$
\liminf _{n \rightarrow \infty} \sum_{s=n}^{n+\delta_{2}-1} \psi_{s+\delta_{2}}>\left(\frac{\delta_{2}}{\delta_{2}+1}\right)^{\delta_{2}+1}
$$


then every solution of Equation $\left(E_{+}\right)$is oscillatory.

Proof. It is known (see [7]) that condition (2.27) is sufficient for inequality (2.25) to have no eventually negative solution. On the other hand, condition (2.28) is sufficient for inequality (2.26) to have no eventually positive solution.

Remark 2.6. From the results presented in this section, we observe that when the coefficient $p_{n}=0$ or the condition on $\left\{p_{n}\right\}$ is violated the conclusion of the theorem may be replaced by "Every solution $\left\{x_{n}\right\}$ of equation $\left(E_{ \pm}\right)$is oscillatory or $x_{n} \rightarrow \infty$ as $n \rightarrow \infty$ ".

Once again from the proofs, we see that if $q_{n}=0$ or condition on $\left\{q_{n}\right\}$ is violated then the conclusion of the theorems may be replaced by "Every solution $\left\{x_{n}\right\}$ of Equation $\left(E_{ \pm}\right)$ is oscillatory or $x_{n} \rightarrow 0$ as $n \rightarrow \infty$ ".

\section{Examples}

In this section, we provide some examples to illustrate the main results.

Example 1. Consider the difference equation

$$
\Delta^{2}\left(x_{n}+2^{\tau_{1}} x_{n-\tau_{1}}-2^{-\tau_{2}} x_{n+\tau_{2}}\right)=\frac{1}{2}\left(2^{\sigma_{1}} x_{n-\sigma_{1}}+2^{-\sigma_{2}} x_{n+\sigma_{2}}\right) .
$$

Here $\alpha=\beta=1, a=2^{\tau_{1}}, \quad b=2^{-\tau_{2}}, q_{n}=2^{\sigma_{1}-1}, p_{n}=2^{-\sigma_{2}-1}$.

It is easy to see that condition (2.9) of Theorem 2.3 is not satisfied and hence Equation (3.1) has a nonoscillatory solution $\left\{x_{n}\right\}=\left\{2^{n}\right\} \rightarrow \infty$ as $n \rightarrow \infty$.

Example 2. Consider the difference equation

$$
\Delta^{2}\left(x_{n}+2^{-\tau_{1}} x_{n-\tau_{1}}-2^{\tau_{2}} x_{n+\tau_{2}}\right)=\frac{1}{8}\left(2^{-\sigma_{1}} x_{n-\sigma_{1}}+2^{\sigma_{2}} x_{n+\sigma_{2}}\right) .
$$

Here $\alpha=\beta=1, a=2^{-\tau_{1}}, \quad b=2^{\tau_{2}}, q_{n}=2^{-\sigma_{1}-3}, p_{n}=2^{\sigma_{2}-3}, \sigma_{1}>\tau_{1}, \sigma_{2}>\tau_{2}$. It is easy to see that condition (2.10) of Theorem 2.3 is not satisfied and hence Equation

(3.2) has a nonoscillatory solution $\left\{x_{n}\right\}=\left\{\frac{1}{2^{n}}\right\} \rightarrow 0$ as

$n \rightarrow \infty$.

Example 3. Consider the difference equation

$$
\Delta^{2}\left[\left(x_{n}+a x_{n-\tau_{1}}+b x_{n-\tau_{2}}\right)^{\alpha}\right]=q x_{n-\sigma_{1}}^{\alpha}+p x_{n+\sigma_{2}}^{\alpha} \quad n \geq n_{0} .
$$

where $p>0, q>0$ are constants and $\sigma_{1}>\tau_{1}$ and $\sigma_{2}>\tau_{2}$. It is easy to see that

$$
\begin{aligned}
& p_{n}^{*}=p \text { and } p_{n}^{*}=p \text {. Assume that } \epsilon>0 \text {. Let } \phi_{n}=\left(\frac{2+\varepsilon}{2}\right)\left(\frac{\delta_{1}^{\delta_{1}}}{\left(\delta_{1}+1\right)^{\delta_{1}+1}}\right), \\
& \psi_{n}=\left(\frac{2+\varepsilon}{2}\right)\left(\frac{\delta_{2}^{\delta_{2}}}{\left(\delta_{2}+1\right)^{\delta_{2}+1}}\right) \text {, where } \delta_{i}=\frac{\sigma_{i}-\tau_{i}}{2}, i=1,2 .
\end{aligned}
$$

Clearly $\left\{\varphi_{n}\right\}$ and $\left\{\psi_{n}\right\}$ satisfies the condition of Corollary 2.6. If

$$
q>4^{\alpha-1}\left(\frac{2+\varepsilon}{2}\right)^{2}\left(\frac{\delta_{1}^{\delta_{1}}}{\left(\delta_{1}+1\right)^{\delta_{1}+1}}\right)^{2}\left(1+a^{\alpha}+\frac{b^{\alpha}}{2^{\alpha-1}}\right)
$$


and

$$
p>4^{\alpha-1}\left(\frac{2+\varepsilon}{2}\right)^{2}\left(\frac{\delta_{2}^{\delta_{2}}}{\left(\delta_{2}+1\right)^{\delta_{2}+1}}\right)^{2}\left(1+a^{\alpha}+\frac{b^{\alpha}}{2^{\alpha-1}}\right)
$$

then condition (2.23) and (2.24) holds. Further we see that

$$
\liminf _{n \rightarrow \infty} \sum_{s=n-\delta_{1}}^{n-1} \phi_{s-\delta_{1}}=\left(\frac{2+\varepsilon}{2}\right) \frac{\delta_{1}^{\delta_{1}}}{\left(\delta_{1}+1\right)^{\delta_{1}+1}} \delta_{1}>\left(\frac{\delta_{1}}{\delta_{1}+1}\right)^{\delta_{1}+1}
$$

and

$$
\liminf _{n \rightarrow \infty} \sum_{s=n}^{n+\delta_{2}-1} \psi_{s+\delta_{2}}=\left(\frac{2+\varepsilon}{2}\right) \frac{\delta_{2}^{\delta_{2}}}{\left(\delta_{2}+1\right)^{\delta_{2}+1}} \delta_{2}>\left(\frac{\delta_{2}}{\delta_{2}+1}\right)^{\delta_{2}+1}
$$

By Corollary 2.6, we see that all solutions of Equation (3.3) are oscillatory.

We conclude this article with the following remark.

Remark 3.1. It would be interesting to extend the results of this article to the equation

$$
\Delta\left(a_{n} \Delta\left(x_{n}+b x_{n-\tau_{1}} \pm c x_{n+\tau_{2}}\right)^{\alpha}\right)=q_{n} x_{n-\sigma_{1}}^{\beta}+p_{n} x_{n+\sigma_{2}}^{\gamma}
$$

where $\alpha, \beta$, and $\gamma$ are ratio of odd positive integers.

\section{Author details}

${ }^{1}$ Ramanujan Institute for Advanced Study in Mathematics, University of Madras, Chennai 600 005, India ${ }^{2}$ Departamento de Matemática, Universidade dos Açores, Ponta Delgada, Portugal

\section{Authors' contributions}

ET framed the problem and NK solved the problem. SP modified and made necessary changes in the proof of the theorems. All authors read and approved the manuscript.

\section{Competing interests}

The authors declare that they have no competing interests.

Received: 3 October 2011 Accepted: 27 January 2012 Published: 27 January 2012

\section{References}

1. Agarwal, RP: Difference Equations and Inequalities. Marcel Dekkar, New York, 2 (2000)

2. Agarwal, RP, Bohner, M, Grace, SR, O'Regan, D: Discrete Oscillation Theory. Hindawi Publ. Corporation, New York (2005)

3. Agarwal, RP, Grace, SR: Oscillation theorems for certain difference equations. pp. 299-308. Dyna. Syst. Appl 9, (2000)

4. Migda, M, Migda, J: Oscillatory and asymptotic properties of solutions of even order neutral difference equations. J Diff Equ Appl. 15, 1077-1084 (2009). doi:10.1080/10236190903032708

5. Agarwal, RP, Grace, SR: The oscillation of certain difference equations. Math Comput Model. 30, 77-83 (1999). doi:10.1016/50895-7177(99)00199-5

6. Grace, SR: Oscillation of certain difference equations of mixed type. J Math Anal Appl. 224, 241-254 (1998). doi:10.1006/ jmaa.1998.6001

7. Gyori, I, Laddas, G: Oscillation Theory of Delay Differential Equations. Clarendon Press, Oxford (1991)

8. Jiang, J: Oscillation of second order nonlinear neutral delay difference equations. Appl Math Comput. 146, 791-801 (2003). doi:10.1016/50096-3003(02)00631-8

9. Hardy, GH, Littlewood, JE, Polya, G: Inequalities. Cambridge Univ Press, Cambridge, 2 (1998) 